\title{
Congenital Atresia of the Left Main Coronary Artery Associated With Patent Ductus Arteriosus and Aortic Regurgitation
}

\author{
Hsin-Hua Chou, MD; Chih-Hsiang Chan, MD**; Kuei-Ton Tsai, MD*; \\ Chung-I Chang, $\mathrm{MD}^{\dagger}$; Yu-Lin Ko, MD, $\mathrm{PhD}$
}

\begin{abstract}
Congenital left main coronary artery (LMCA) atresia is a rare anomaly and surgical revascularization is recommended once the diagnosis is confirmed. In some cases, LMCA atresia will coexist with other cardiac anomalies, mostly in pediatric patients. A 32-year-old woman had congenital LMCA atresia associated with patent ductus arteriosus (PDA), aortic regurgitation (AR), and moderate pulmonary hypertension. The PDA and AR were repaired surgically and the patient's symptoms improved. Coronary revascularization was not performed because the LCA was supplied by 2 large conus arteries, as "naturally occurring" bypass arteries. The patient remained asymptomatic at the 2.5-year follow-up visit. Given the positive outcome in this patient, surgical repair of the associated cardiac defect without coronary revascularization may be a reasonable approach for patients with congenital LMCA atresia, in which the left coronary arterial tree is supplied by other "naturally occurring" bypass arteries. (Circ J 2009; 73: 1163-1166)
\end{abstract}

Key Words: Angiography; Congenital heart disease; Coronary heart disease; Valvuloplasty

C ongenital atresia of the left main coronary artery (LMCA) is an extremely rare anomaly, with fewer than 40 cases reported in the literature. Despite its rare incidence, it has an unfavorable outcome because it inevitably results in severe myocardial ischemia, myocardial infarction, and even sudden cardiac death if left untreated! In infants and children this anomaly may be associated with other cardiac defects, such as supravalvular aortic stenosis, 2,3 ventricular septal defect and pulmonary stenosis, right coronary ostium stenosis, 5 and prolapse of the mitral valve. Because of the poor clinical outcome of this disorder, surgical revascularization of LMCA atresia is mostly recommended, regardless of the presence of associated cardiac anomalies.

\section{Case Report}

A 32-year-old woman visited hospital with the chief complaint of respiratory distress for the past 2 years. Although an abnormal cardiac murmur had been detected in childhood, she had only experienced mild exercise intolerance until early 2004, when she developed dyspnea on exertion, as well as paroxysmal nocturnal dyspnea and progres-

(Received March 31, 2008; revised manuscript received July 9, 2008; accepted July 15, 2008; released online December 19, 2008)

Division of Cardiology, Department of Internal Medicine, *Division of Cardiovascular Surgery, Department of Surgery, Buddhist Tzu Chi General Hospital, Taipei Branch, **Division of Cardiovascular Surgery, Department of Surgery, Min-Sheng General Hospital and tDivision of Cardiovascular Surgery, Department of Surgery, National Taiwan University Hospital, Taipei, Taiwan

Mailing address: Yu-Lin Ko, MD, PhD, Division of Cardiology, Department of Internal Medicine, Buddhist Tzu Chi General Hospital, Taipei Branch, 289 Jianguo Road, Xindian City, Taipei 231, Taiwan, ROC. E-mail: yulinkotw@yahoo.com.tw

All rights are reserved to the Japanese Circulation Society. For permissions, please e-mail: cj@j-circ.or.jp sive orthopnea. She had been seen at another hospital where congestive heart failure was diagnosed. Digitalis and diuretics were administered, but she remained symptomatic.

In the present admission to hospital in October 2005, the patient had symptoms of overt heart failure. Physical examination revealed a $3 / 6$ continuous murmur at the left upper sternal border. Transthoracic echocardiography demonstrated a dilated left atrium and left ventricle with impaired left ventricular contractility. Moderate aortic regurgitation (AR) and moderate pulmonary hypertension were also detected, but no intracardiac shunt was seen on 2-dimensional echocardiography. The results of subsequent transesophageal echocardiography confirmed the transthoracic echocardiographic findings, apart from revealing a patent ductus arteriosus (PDA) and a LMCA of abnormal origin between the noncoronary and left coronary sinuses.

Right heart catheterization disclosed a 13\% increment in oxygen saturation at the level of the main pulmonary artery. The main pulmonary arterial pressure was $57 \mathrm{mmHg}$. The ratio of pulmonary to systemic blood flow $(\mathrm{Qp} / \mathrm{Qs})$ was 1.76. Aortography at the level of the thoracic descending aorta showed a large PDA connecting the descending aorta and main pulmonary artery, with a left-to-right shunt. Aortic root angiography demonstrated grade 3/4 AR. During coronary arteriography, the LMCA was not opacified, even after direct injection of contrast medium into the left sinus of Valsalva. Two large conus arteries originating from a separate ostium alongside the orifice of the right coronary artery (RCA) were revealed using a right Judkins technique. These 2 arteries connected to the middle portion of the left anterior descending artery (LAD) and perfused the entire left coronary arterial tree in an antegrade and retrograde fashion (Figure 1A). The RCA had a normal caliber and filled the LAD and left circumflex artery (LCx) via numerous small collateral vessels from the posterior descending artery (Figure 1B). The ostium of the LMCA formed a blind loop 

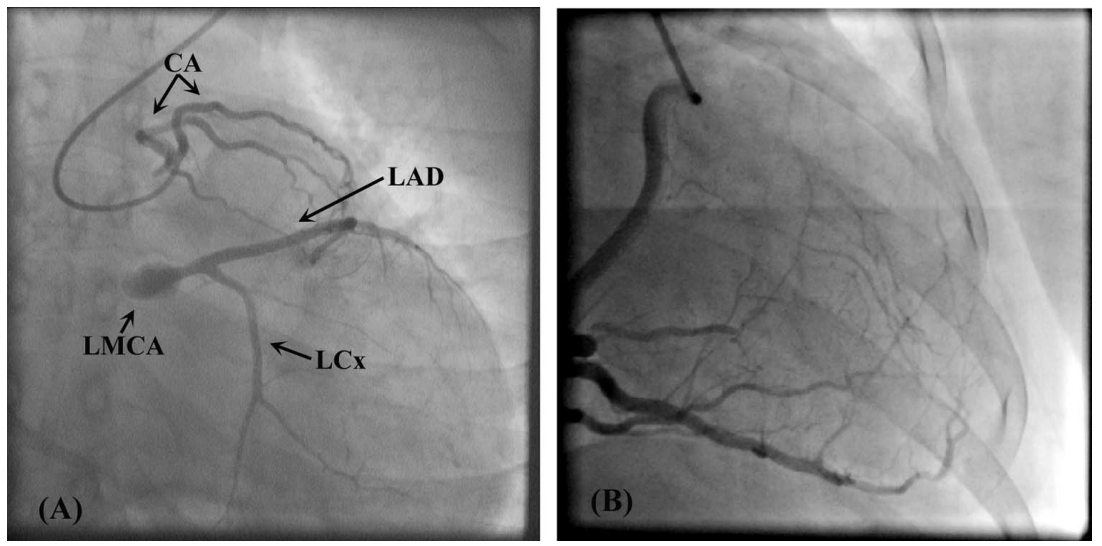

Figure 1. (A) Two large conus arteries (CA) originate from a separate ostium alongside the orifice of the right coronary artery (RCA) and connect to the middle portion of the left anterior descending artery (LAD). The left main coronary artery (LMCA) appears as a blind loop. (B) Right coronary arteriogram shows the normal RCA with numerous small collateral vessels from the posterior descending artery to the left coronary arteries. LCx, left circumflex artery.

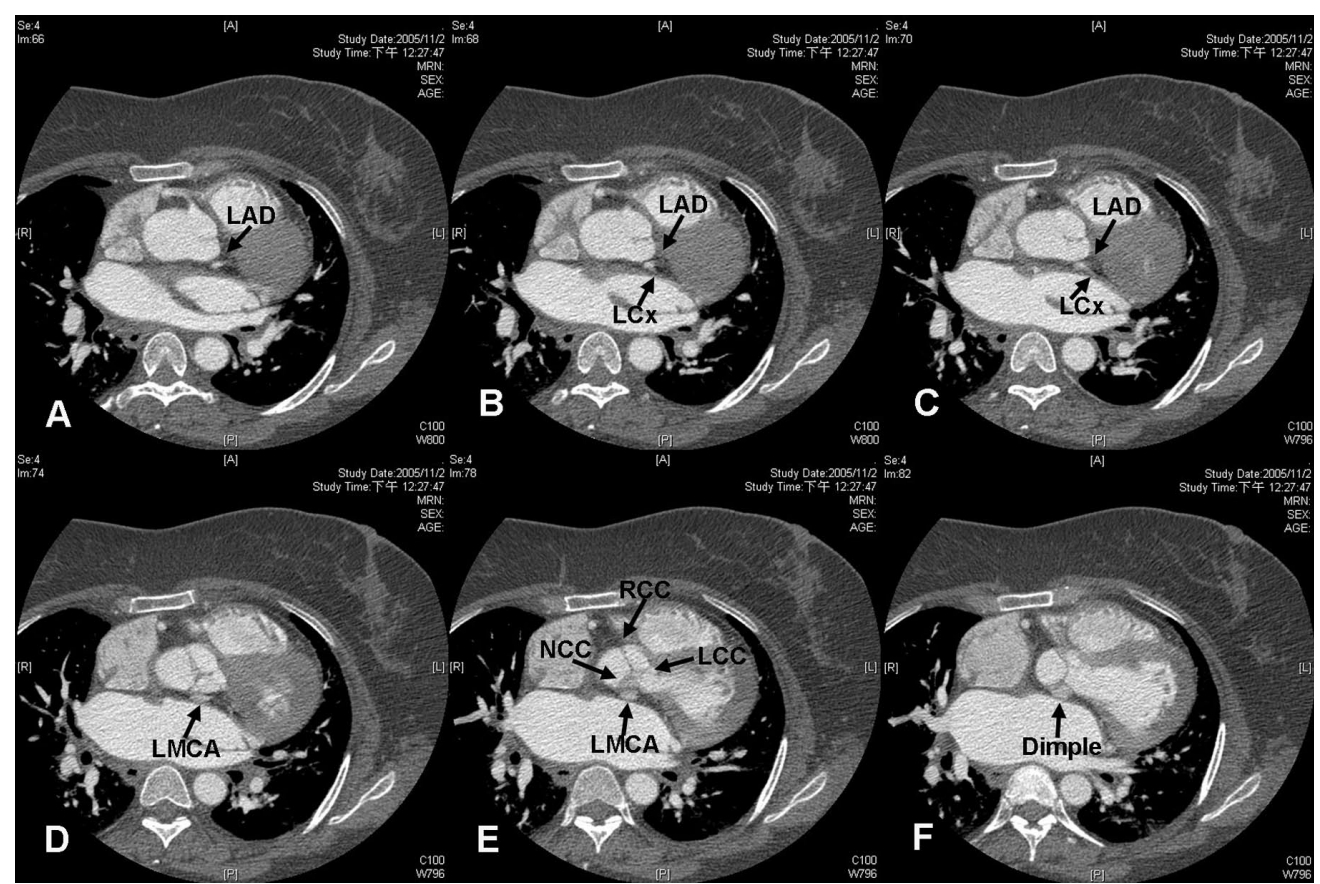

Figure 2. (A-F) Left anterior descending artery (LAD), left circumflex artery (LCx) and the left main coronary artery (LMCA) can be traced retrograde to the dimple located in the subaortic area. RCC, right coronary cusp; LCC, left coronary cusp; NCC, noncoronary cusp.

with fusiform dilatation that did not connect with the aortic root. Computed tomographic angiograms of the coronary arteries demonstrated that the fusiform dilatation of the LMCA ostium formed a dimple at the subaortic area (Figure 2). Preoperative technetium-99 scintigraphy using intravenous dipyridamole revealed perfusion defects in the anterior region and the adjacent anterolateral wall of the left ventricle (Figure 3A).

The patient was referred for surgery in December 2005. During the operation, a 6-mm PDA was confirmed, with the pulmonary part of the ductus vessel located at the cephalic portion of the bifurcation of the main pulmonary artery. Only the RCA orifice was evident. A 6-mm dimple in the subaortic area, located below and between the noncoronary and left coronary sinus of Valsalva, hindered the normal coaptation of the aortic valve (Figure 4). Two large conus arteries originated from a separate orifice beside the RCA orifice, and coursed anteriorly and entered the middle part of the LAD. PDA obliteration through a main pulmonary artery approach and aortic valve repair with partial approximation of the noncoronary and left coronary cusps (LCCs) were performed. Because the patient had the 2 large conus arteries originating from the aorta and bypassing the LMCA, and was free from anginal symptoms, surgical revascularization was not performed.

The patient had an uneventful recovery. Follow-up 2dimensional echocardiography demonstrated a reduction in the size of the left atrium and left ventricle, with improved left ventricular contractility. The AR resolved, and the pulmonary arterial pressure returned to normal limits. Technetium-99 stress myocardial perfusion scintigraphy, performed 6 months (Figure 3B) and 2.5 years (Figure 3C) postoperatively, showed significant improvement of the perfusion defect in the anterior wall of the left ventricle. The patient remained free from heart failure or anginal symptoms at the 2.5-year follow-up visit. 

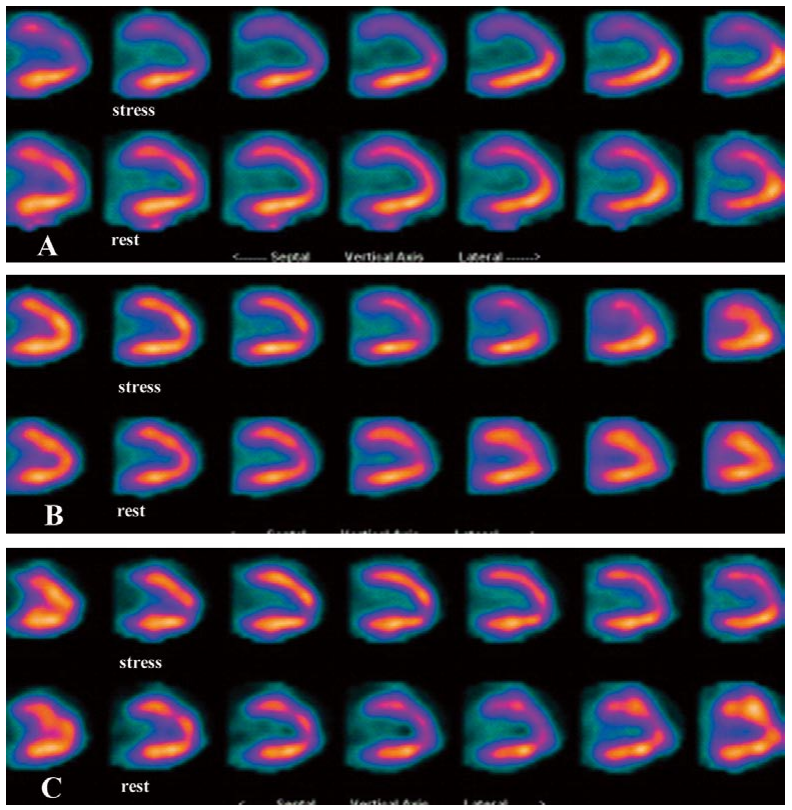

Figure 3. Preoperative technetium-99 scintigraphy (A) reveals a perfusion defect in the anterior region of the left ventricle. Postoperative scintigraphy performed at 6 months $(\mathbf{B})$ and at 2.5 years $(\mathbf{C})$ demonstrate significant improvement of the perfusion defect in the anterior wall of the left ventricle.

\section{Discussion}

Congenital atresia of the LMCA is a very rare anomaly, with only 39 cases reported at the time of writing. It was originally regarded as a variant of the single coronary artery until the mid 1970s, when Lurie first defined this anomaly as a distinct entity with a characteristic flow pattern and physiology6 The clinical outcomes for these 2 congenital anomalies are also different. The single coronary artery is usually considered a benign anomaly that does not cause symptoms and is compatible with a normal life span; it is often diagnosed only at autopsy? However, patients with congenital LMCA atresia often present with angina, myocardial infarction, failure to thrive or sudden cardiac death!

In infants and children, LMCA atresia may be associated with other cardiac defects. In the 28 cases reviewed by Musiani et al, 5 pediatric patients had other cardiac defects! Interestingly, no associated cardiac anomaly has been reported in any adult patient with LMCA atresia. It is likely that the coexistence of cardiac defects results in either increasing myocardial oxygen demand or decreasing myocardial oxygen supply, which causes symptoms early in life. The present patient had congenital LMCA atresia associated with PDA and AR, a diagnosis not previously reported in the literature. PDA increases myocardial oxygen demand by increasing left ventricular preload, and thus exacerbates the degree of myocardial ischemia in patients with congenital LMCA atresia. Furthermore, the LMCA in the present patient originated from a dimple located in the subvalvular area between the noncoronary and LCCs, which impaired the normal coaptation of the aortic valve and resulted in AR. The AR further exacerbated the myocardial ischemia. AR repair and PDA closure improved coronary blood supply and decreased myocardial oxygen demand because of the reduction in left ventricular preload.

The long-term result of coronary artery bypass grafting

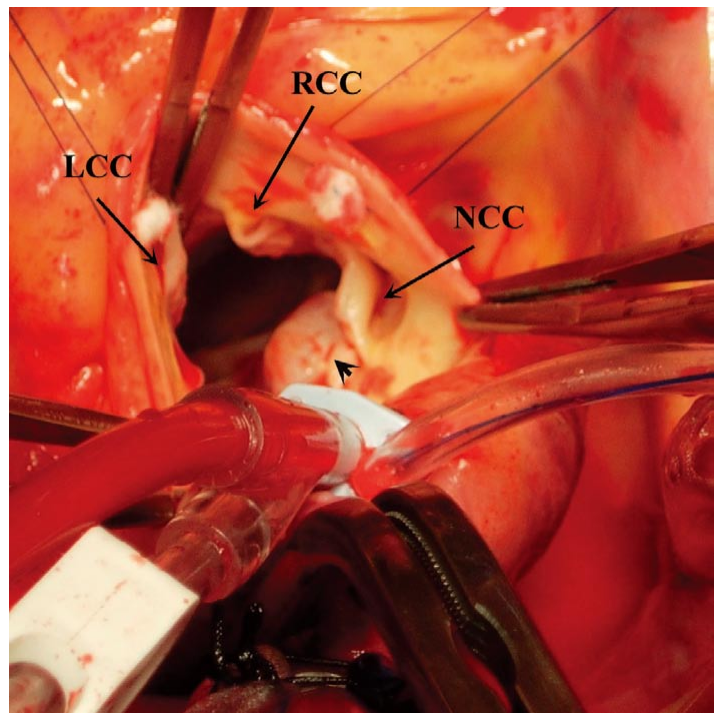

Figure 4. Intraoperative image after aortotomy showing the subvalvular dimple (arrowhead) between the noncoronary cusp (NCC) and the left coronary cusp (LCC), which hinders the normal coaptation of the aortic valve and results in aortic regurgitation. RCC, right coronary cusp.

in pediatric patients is questionable, especially if saphenous vein grafts are used; however, coronary artery revascularization is the procedure of choice in adult patients with congenital LMCA atresia, because internal mammary artery (IMA) grafts have superior long-term patency! However, Sato et al used an IMA graft to the LAD in an 18-year-old male patient and found that the IMA flow was insufficient in the postoperative study performed 8 months after surgery? Ghosh et al describe a 55-year-old man with isolated congenital LMCA atresia and coexistent 2-vessel atherosclerosis? Although there were clinically significant atherosclerotic lesions of the proximal LAD, the origin of the $\mathrm{LCx}$ and the first diagonal branch, they only revascularized the diagonal and marginal arteries because the LAD was supplied by a large conus artery originating from the aorta. Elian et al also reported a 42-year-old male patient with congenital LMCA atresia in whom the left coronary arteries were supplied via 2 large vessels from the posterior descending branch and the posterolateral branch of the RCA 10 Although thallium stress scintigraphy demonstrated reversible perfusion defect at the septum and the anterolateral wall, the authors did not recommend revascularization for that patient because he was completely asymptomatic and remained asymptomatic 18 months post catheterization. Because the present patient was free from angina symptoms, and had 2 large conus branches connected to the LAD as "naturally occurring" bypass arteries, surgical revascularization was not performed. To our knowledge, this is the first reported case of congenital LMCA atresia associated with PDA and clinically significant AR. Given the positive outcome in this patient, surgical repair of the associated cardiac defect without coronary revascularization may be a reasonable approach for patients with congenital LMCA atresia, in which the left coronary arterial tree is supplied by other "naturally occurring" bypass arteries.

\section{References}

1. Musiani A, Cernigliaro C, Sansa M, Maselli D, De Gasperis C. Left main coronary artery atresia: Literature review and therapeutical 
considerations. Eur J Cardiothorac Surg 1997; 11: 505-514.

2. Allen HD, Moller JH, Formanek A, Nicoloff D. Atresia of the proximal left coronary artery associated with supravalvular aortic stenosis: Surgical treatment. Thorac Cardiovasc Surg 1974; 67: 266-271.

3. Rosenkranz ER, Murphy DJ Jr, Cosgrove DM 3rd. Surgical management of left coronary artery ostial atresia and supravalvar aortic stenosis. Ann Thorac Surg 1992; 54: 779-781.

4. Vidne BA, Nili M, Aygen M, Levy MJ. Congenital atresia of the left main coronary artery ostium. Scand Cardiovasc J 1979; 13: 37-40.

5. Goormaghtigh N, De Vos L, Blancquaert A. Ostial stenosis of coronary arteries in nine-year-old girl. Arch Intern Med 1955; 95: 341 348.

6. Lurie PR. Abnormalitis and diseases of the coronary vessels. In: Moss AJ, Adams FH, Emmanouilides GC, eds. Heart disease in infants, children, and adolescents, 2nd edn. Baltimore: Williams \& Wilkins, 1977.

7. Murphy ML. Single coronary artery. Am Heart J 1967; 74: 557-561.

8. Sato S, Majima T, Kawaguchi T, Kitagawa S, Takano H, Ihara K. Congenital atresia of the left main coronary artery ostium: A case suffering from ventricular tachycardia. Nippon Kyobu Geka Gakkai Zasshi 1990; 38: 1467-1474 (in Japanese with English abstract).

9. Ghosh PK, Friedman M, Vidne BA. Isolated congenital atresia of the left main coronary artery and atherosclerosis. Ann Thorac Surg 1993; 55: $1564-1565$

10. Elian D, Hegesh J, Agranat O, Guetta V, Har-Zahav Y, Rath S, et al. Left main coronary artery atresia: Extremely rare coronary anomaly in an asymptomatic adult and an adolescent soccer player. Cardiol Rev 2003; 11: 160-162. 